\title{
The Edge of the Milky Way Stellar Disk Revealed Using Clump Giant Stars as Distance Indicators
}

\author{
D. Minniti ${ }^{1,2,3}$, R. K. Saito ${ }^{1}$, J. Alonso-García ${ }^{1}$, P. W. Lucas $^{4}$ and M. Hempel ${ }^{1}$ \\ ${ }^{1}$ Departamento de Astronomía y Astrofísica, Pontificia Universidad Católica de Chile, \\ Vicuña Mackenna 4860, Casilla 306, Santiago 22, Chile \\ ${ }^{2}$ Vatican Observatory, Vatican City State V-00120, Italy \\ ${ }^{3}$ European Southern Observatory, Vitacura 3107, Santiago, Chile \\ ${ }^{4}$ Centre for Astrophysics Research, University of Hertfordshire, College Lane, Hatfield \\ AL10 9AB, UK
}

\begin{abstract}
We use the clump giants of the disk as standard candles calibrated from Hipparcos parallaxes in order to map their distribution with two new near-IR surveys of the Galactic plane: UKIDSS-GPS and VVV. We explore different selection cuts of clump giants. We conclude that there is an edge of the stellar disk of the Milky Way at $R=13.9 \pm 0.5 \mathrm{kpc}$ along various lines of sight across the galaxy. The effect of the warp is considered, taking fields at different longitudes and above and below the plane. We demonstrate that the edge of the stellar disk of the Milky Way can now be mapped in the near infrared in order to test different models, and to establish our own place within the galaxy.
\end{abstract}

Subject headings: Galaxy: disk — Galaxy: structure — Stars: distances — Stars: late-type

\section{Introduction}

Most spiral galaxies have rather sharp edges in their stellar disks (van der Kruit 1979, 2007). The situation for the Milky Way is unclear. The disk of our galaxy is well represented by an exponential density profile (Freeman 1970), with some evidence for a stellar disk cut-off at a radius of $\sim 14 \mathrm{kpc}$ towards the anticentre region (Robin et al. 1992), but other claims for a warped and flaring stellar disk out to $23 \mathrm{kpc}$ (Momany et al. 2006). Based upon the scaling relation established by other galaxies, the radial cut off should be between 10 and $25 \mathrm{kpc}$ (Pohlen et al. 2004; Kregel \& van der Kruit 2004). Here we report observations of "clump red giant" stars, which are good distance indicators (Alves 2000; Paczynski \& Stanek 
1998), along 10 lines of sight in the disk of the Milky Way. The data reveal an edge to the disk at $R=13.9 \pm 0.6 \mathrm{kpc}$. Why there should be a sharp edge to the stars, while the gas profile is much more extended and does not show such an edge (Kalberla \& Kerp 2009), remains unclear, but a critical test would be the measurement of the gas and star density in situ (Kennicutt 1989).

There are two new deep surveys of the Milky Way disk in the near-infrared: the UKIDSS Galactic Plane Survey (GPS), that is mapping the Northern disk (Lucas et al. 2008), and the VISTA Variables in the Vía Láctea (VVV) Survey, that is mapping 520 square degrees in the Southern disk and bulge of our Galaxy (Minniti et al. 2010). While previous observations in the Galactic plane have been limited by crowding, source dimness, and interstellar extinction, these new public surveys allow us to pierce through the whole disk, reaching red giants in the horizontal branch (clump giants) located at the other side of our galaxy for the first time in different near-IR pass-bands. The VVV survey observes in the $Z$ (0.87 microns), $Y$ (1.02 microns), $J$ (1.25 microns), $H$ (1.64 microns), and $K_{\mathrm{s}}$ (2.14 microns) bands, while the UKIDSS-GPS is observing in the $J$ (1.25 microns), $H$ (1.64 microns), and $K$ (2.14 microns) bands; with the photometry uniformly calibrated in the 2MASS system (Skrutskie et al. 2006).

Clump giants are excellent tools to study the structure of our galaxy because of two main reasons, discussed below: (i) they are good distance indicators, and (ii) they are good tracers of the mass in the disks of galaxies. The UKIDSS-GPS and VVV Surveys give the largest homogeneous census of clump giants out to well beyond the extent of the Milky Way stellar disk. The limiting DoPhot (Schechter et al. 1993) magnitude of single epoch VVV images processed by the Cambridge Astronomical Survey Unit (CASU) pipeline v1.0 is $K_{\mathrm{s}}=17.5$ in the disk fields, and the DoPhot photometry on the combined epochs allow to reach approximately $J=20 \mathrm{mag}$, and $K_{\mathrm{s}}=18.5 \mathrm{mag}$ (Fig. 1), slightly fainter than the UKIDSS-GPS, and much fainter than the near-IR surveys 2MASS and DENIS, for which the limit is $K_{\mathrm{s}}=14.3 \mathrm{mag}$ (Skrutskie et al. 2006). The distance probed along the line of sight depends on the reddening of the fields. For example, in disk fields with low extinction $\left(A_{\mathrm{V}}<3 \mathrm{mag}\right)$ the UKIDSS-GPS and VVV surveys would see clump giants beyond $50 \mathrm{kpc}$. Therefore we can search for the edge of the disk of our galaxy using well calibrated standard candles.

\section{Observational Data}

The clump giants with known parallaxes measured by the Hipparcos satellite are the best collectively calibrated standard candles (Paczynski \& Stanek 1998). 

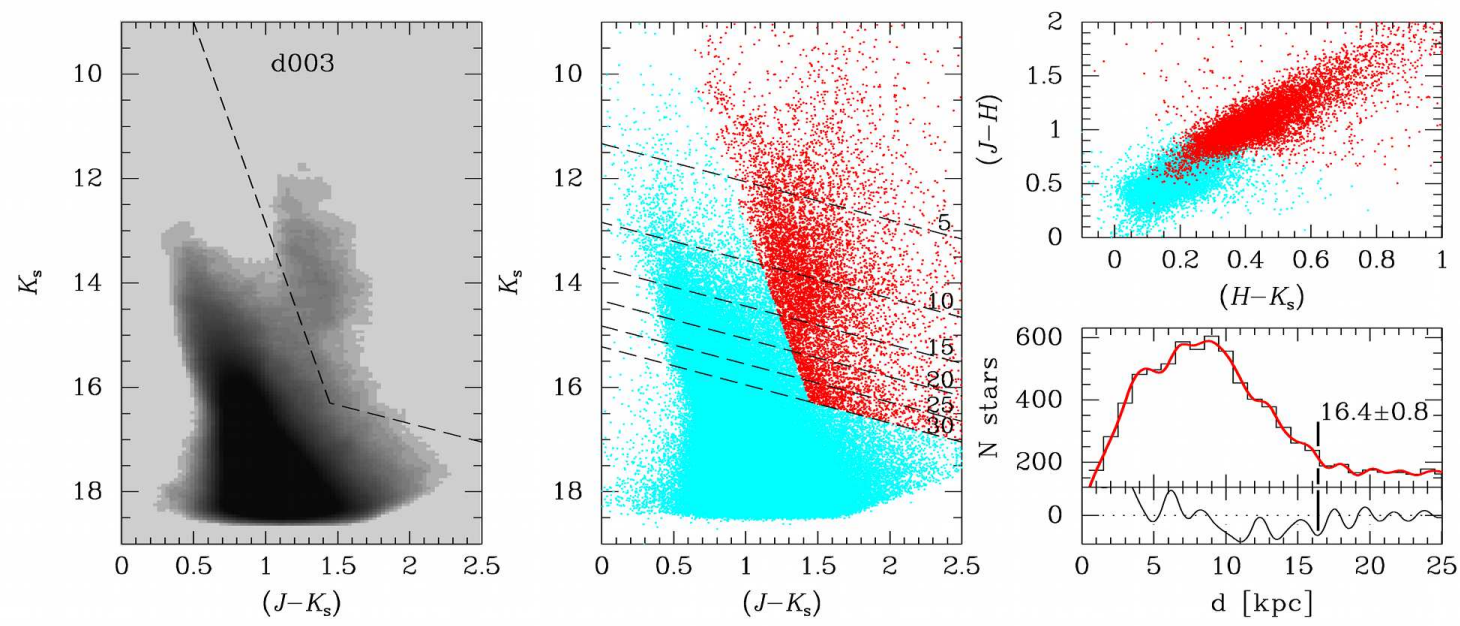

Fig. 1.- Selection of giant stars used in the distance calculation. The left-hand panel shows the colour-magnitude diagram (CMD) of VVV field d003 as a density map. The adopted color-cut is shown, which is similar to the cut for other fields, even though the location of the edge feature is relatively insensitive to these cuts. The CMD in the central panel and the colour-colour diagram in the top-right panel show in dark gray the selection of giant stars used in the distance calculation. Dashed lines in the CMD mark the distance in kpc for the selected stars according with equation 2. The bottom-right panel shows the distance distribution and respective derivative curve, with the outermost the minimum derivative marked.

Figure 1 shows a colour-colour and colour-diagram magnitude for the VVV field d003. In order to select the giant stars a colour cut was applied in the $K_{\mathrm{s}} v s$. $\left(J-K_{\mathrm{s}}\right)$, as shown in left-hand and central panels of Fig. 1. Not all selected stars are clump giants, but the presence of sub-giant branch and red-giant branch stars, or even main-sequence stars at fainter magnitudes, contribute to the smooth underlying background. These different population do not affect the location of any sharp edges of the stellar distribution, which can however be well defined by the clump giants. The effect of reddening in the selection of clump giants was also tested using different selection criteria both in the colour-magnitude and colour-colour diagrams, finding consistent results.

An accurate $K$-band calibration of the red clump giant luminosity was obtained and applied to the red giant clump of the Galactic bulge (Alves 2000), and of the Large Magellanic Cloud (Alves et al. 2002). Our magnitudes are in the 2MASS magnitude system, and we transformed the $K$-band magnitudes of clump giants of Hipparcos (Alves 2000) to $K_{\mathrm{s}}$ 
magnitudes using $K=K_{\mathrm{s}}+0.044$. The resulting zero point differences should be less than 0.02 magnitudes (Alves et al. 2002). For the Ks photometry, the distance modulus to a red clump giant in the outer disk would be:

$$
\mu=K_{\mathrm{s}}-\frac{A_{K_{\mathrm{s}}}}{\left(A_{J}-A_{K_{\mathrm{s}}}\right)}\left[\left(J-K_{\mathrm{s}}\right)-\left(J-K_{\mathrm{s}}\right)_{0}\right]-M_{K_{\mathrm{s}}}
$$

where we adopted $A_{K_{\mathrm{s}}} /\left(A_{J}-A_{K_{\mathrm{s}}}\right)=0.73,\left(J-K_{\mathrm{s}}\right)_{0}=0.70 \pm 0.05$ and $M_{K_{\mathrm{c}}}=-1.65 \pm 0.03$ as the mean values for the red clump giants of the Milky Way disk (Alves et al. 2002). There should be negligible metallicity dependence of these mean values because we are looking at a stellar population of the Milky Way disk that should be similar to that of the Solar neighbourhood where Hipparcos distances of clump giants were calibrated. Adopting these means magnitudes and colours, and the reddening coefficients (Cardelli et al. 1989) yields:

$$
\mu=-5+5 \log d(\mathrm{pc})=K_{\mathrm{s}}-0.73\left(J-K_{\mathrm{s}}\right)+2.16 .
$$
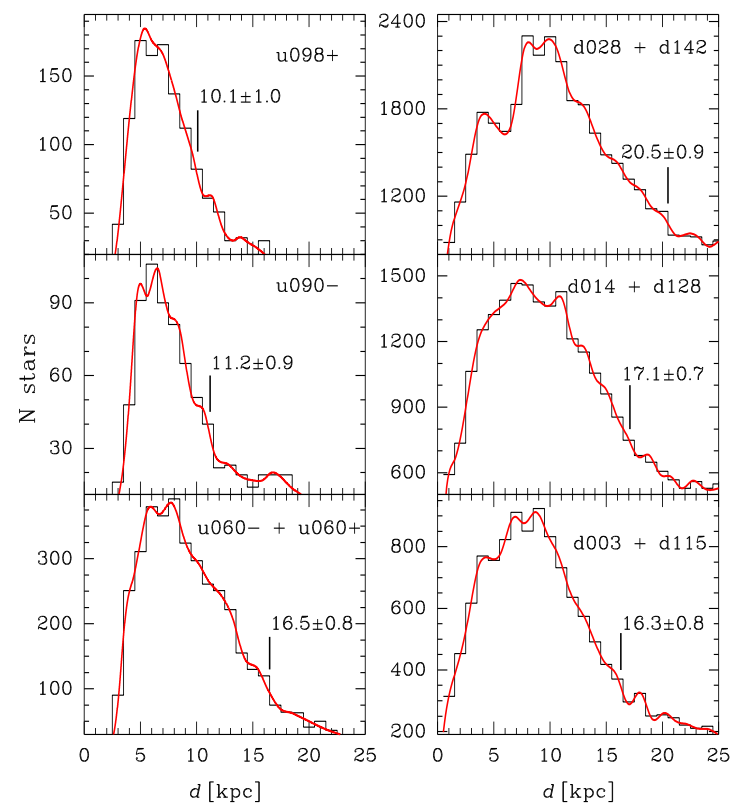

Fig. 2.- Distance distribution for clump giant stars along different lines of sight. The distance measured to the edge of the Milky Way stellar disk is labelled for each field. The thin solid line is the smoothed distribution. These stellar distributions are rich with information about the disk structure. For example, we discarded the presence of distant star clusters, where the red giant clump could mask an edge feature, but see some over-density fluctuations that may be due to spiral arms.

Using this equation we computed the distance modulus (and distance in $\mathrm{kpc}$ ) for every single clump giant candidate in the fields. For the UKIDSS-GPS $K$-band data the com- 
putations are the same, but with $A_{K} /\left(A_{J}-A_{K}\right)=0.68,(J-K)_{0}=0.66 \pm 0.04$ and $M_{K}=-1.61 \pm 0.03$.

In order to determine the disk edge we computed the distribution in distance for the clump giants in each field. Thus we coadded the distance distribution of fields located above and below the plane at same Galactic longitude. The coadded distance distribution for each longitude was analyzed non-parametrically using the local likelihood density estimation method (Loader 1996). Finally, the derivative method was applied for the distribution curve. This method is simple and robust, and can be used to detect the structures in the distance distribution. We defined as the disk edge the outermost point of minimum derivative in each case. The uncertainties were calculated with a Monte Carlo procedure, assuming Poisson errors.

The uncertainties due to the photometric errors do not produce any trend in the calculations and are much smaller than the statistical error. The resulting distance distributions along different lines of sight are shown in Fig. 2. In all fields there is a density drop in the clump giant distribution at a corresponding Galactocentric distance of about $13.9 \mathrm{kpc}$ (assuming $R_{o}=8 \mathrm{kpc}$ ), as listed in Table 1,

We first noticed the sharp termination of the clump giant distribution in the colormagnitude diagram of the VVV field $\mathrm{d} 001\left(l, b=295.4^{\circ},-1.7^{\circ}\right)$, a very heavily reddened area located in the outskirts of the Carina star forming region (Saito et al. 2010). Similar behavior is also found in UKIDSS-GPS data for different Galactic longitudes (Lucas et al. 2008).

We studied low-extinction fields across the Milky Way disk (see Table 1). When these were available, we selected pairs of fields located above and below the plane at the same Galactic longitude, in order to account for the Galactic warp, because if the disk is warped, the line of sight can leave the stellar disk before this ends. For example, at $l=300^{\circ}$ the mean warp location is $1^{\circ}$ below the plane (Robin et al. 1992), so the selected VVV field d003 is conveniently located to probe the full extent of the disk. At most other longitudes explored here the warp is absent, with the mean plane being at $b=0^{\circ}$ (Fig. 3). We have also explored a few other UKIDSS-GPS fields located at inner longitudes that yield lower distances. These fields with $l<60^{\circ}$ were discarded because they are heavily reddened and crowded, and the photometry is not as deep as the rest, presumably due to enhanced contamination from the near-side of the inner bar. Systematic errors such as variations of the reddening law have not been included.

It is important to note that the present results are independent of models. They rely on basic assumptions such as that the Hipparcos stellar sample is representative of the entire 
the Milky Way disk, and that clump giants are reliable distance indicators and tracers of the old and intermediate age populations. However, the Besançon Galactic model (Robin et al. 2003 ) with a scale-length of $2.4 \mathrm{kpc}$, including a flaring and warped outer disk, with a disk cutoff at $R=14 \mathrm{kpc}$ seems to reproduce well the observed distance distributions (see Fig,4). Data and model show the same features, with a sharp increase, the peak around $5 \mathrm{kpc}$ and the exponential decrease. The edge is detected in the data and model at the expected place within the errors $[d=10.1 \mathrm{kpc}$ (corresponding to $R=13.9 \mathrm{kpc}$ ), and $d=9.5 \mathrm{kpc}$ $(R=13.4 \mathrm{kpc})$, respectively].

\section{Discussion and Conclusions}

A few studies have discussed the edge of the Milky Way towards the anticentre region previously. The old Galactic disk apparently does not extend beyond $14 \mathrm{kpc}$ on the basis of optical $V$ vs. $(B-V)$ colour-magnitude diagrams and star counts (Robin et al. 1992), which does not contradict the presence of other more distant young stars. Models of the COBE-DIRBE infrared emission maps yield that the edge of the disk is at $4 \mathrm{kpc}$ from the Sun (assuming $R_{o}=8.5 \mathrm{kpc}$, Freudenreich 1996). DENIS star counts reveal the cut off of the stellar disk at a Galactocentric distance of $15 \pm 2 \mathrm{kpc}$ (Ruphy et al. 1996).

In contrast, star counts from 2MASS reveal no radial disk truncation at $14 \mathrm{kpc}$ (Momany et al. 2006). Again, the depth of 2MASS is not enough to reach large distances, which is possible with the VVV and UKIDSS-GPS survey that reach 3-4 magnitudes fainter. More recently, it was found that the 2MASS star counts are best fit if the external disk is truncated at 12-14 kpc (Reylé et al. 2009), while early A-type stars in the anticentre from the IPHAS survey reveal an exponential disk out to $13 \mathrm{kpc}$, with a steeper decline beyond that distance (Sale et al. 2010). Most of the previous evidence for cutoffs in the stellar distribution has been acquired at the anticentre fields. The present deep exploration of different fields in three galactic quadrants finds consistent results.

Even though we interpret this termination of the clump giant distribution as a truncation, we note that a break in the slope of the distribution would also be consistent with the data. It is difficult to measure the sharpness of the cutoff, because the number of stars drop rapidly with distance, but as the surveys progress, we will have more fields in order to explore this issue.

This does not mean that one could not find other young stellar sources beyond the distance that we measure. There are stars detected beyond the edge of the old stellar disk. For example, there is a population of distant young stars at $R=20 \mathrm{kpc}$, in two fields 
Table 1: Selected GPS and VVV fields with relatively low extinction (Schechter et al. 1993) in separate directions across the Milky Way plane.

\begin{tabular}{lcccc} 
Field ID & $l\left[^{\circ}\right]$ & $b\left[^{\circ}\right]$ & $d[\mathrm{kpc}]$ & $R[\mathrm{kpc}]$ \\
\hline GPS-u098+ & 98.4 & +1.0 & $10.1 \pm 1.0$ & $13.9 \pm 1.3$ \\
GPS-u090- & 90.0 & -1.7 & $11.2 \pm 0.9$ & $13.8 \pm 1.1$ \\
GPS-u060- & 60.0 & -2.0 & $16.3 \pm 0.7$ & $14.1 \pm 0.6$ \\
GPS-u060+ & 60.0 & +2.0 & $16.6 \pm 0.8$ & $14.3 \pm 0.7$ \\
VVV-d028 & 334.7 & -1.9 & $20.3 \pm 0.7$ & $13.5 \pm 0.5$ \\
VVV-d142 & 334.8 & +1.9 & $20.7 \pm 1.0$ & $13.8 \pm 0.7$ \\
VVV-d014 & 314.3 & -1.9 & $17.4 \pm 0.9$ & $13.1 \pm 0.6$ \\
VVV-d128 & 314.3 & +1.8 & $16.7 \pm 1.3$ & $12.6 \pm 1.0$ \\
VVV-d003 & 298.3 & -1.9 & $16.4 \pm 0.8$ & $14.6 \pm 0.8$ \\
VVV-d115 & 295.3 & +1.9 & $16.3 \pm 0.8$ & $14.5 \pm 0.7$ \\
\hline
\end{tabular}

located in the third quadrant, slightly above the plane (at $b=7^{\circ}$ and $b=4^{\circ}$ ), based on $U B V$ photometry (Carraro et al. 2010). We see no evidence of a large population of clump giants at that distance in our fields.

$K$-band light traces mass in the disks of spiral galaxies (Rix \& Rieke 1993), and the integrated $K$-band luminosity is dominated by red giants. Thus the clump giants are ideal tracers to define overall features and structural parameters of our Milky Way, like the edge of its disk. The red clump giants trace the old and intermediate age populations, which are more uniformly distributed than young stars, following the mass of the galactic disk. However, thick disk and halo giants are expected to be located beyond the edge of the stellar thin disk, as have been detected by the SEGUE project (de Jong et al. 2010), contributing to the background that we see in all fields beyond a Galactocentric distance of $13.9 \mathrm{kpc}$. A similar result has been recently obtained independently by the GLIMPSE team (Benjamin et al. 2011, in preparation).

Finally, we are able to determine not only the extension but also the shape of the stellar disk of our galaxy for the first time. Asymmetric features such as lopsidedness are common in spiral galaxies (Rix \& Zaritsky 1995). However, the results shown in Fig. 3 suggest that the stellar disk of the Milky Way is not significantly lopsided.

We acknowledge support by the FONDAP Center for Astrophysics, BASAL CATA Cen- 
ter for Astrophysics and Associated Technologies, MILENIO Milky Way Millennium Nucleus from MIDEPLAN, FONDECYT from CONICYT, and the European Southern Observatory. RS acknowledges financial support from CONICYT through GEMINI Project Nr. 32080016. We gratefully acknowledge use of data from the VISTA telescope, and data products from the Cambridge Astronomical Survey Unit, and the Two Micron All Sky Survey (2MASS), which is a joint project of the University of Massachusetts and IPAC/CALTECH, funded by NASA and NSF.

\section{REFERENCES}

Alves, D. R. 2000, ApJ, 539, 732

Alves, D. R., Rejkuba, M., Minniti, D., \& Cook, K. H. 2002, ApJ, 573, L51

Cardelli, J. A., Clayton, G. C., \& Mathis, J. S. 1989, ApJ, 345, 245

Carraro, G., Vázquez, R. A., Costa, E., Perren, G., \& Moitinho, A. 2010, ApJ, 718, 683

Churchwell, E., et al. 2009, PASP, 121, 213

de Jong, J. T. A., Yanny, B., Rix, H.-W., Dolphin, A. E., Martin, N. F., \& Beers, T. C. 2010, ApJ, 714, 663

Freeman, K. C. 1970, ApJ, 160, 811

Freudenreich, H. T. 1996, ApJ, 468, 663

Kalberla, P. M. W., \& Kerp, J. 2009, ARA\&A, 47, 27

Kennicutt, R. C., Jr. 1989, ApJ, 344, 685

Kregel, M., \& van der Kruit, P. C. 2004, MNRAS, 355, 143

Loader, C. R. 1996, The Annals of Statistics, 24, 1602

Lucas, P. W., et al. 2008, MNRAS, 391, 136

Minniti, D., et al. 2010, New Astronomy, 15, 433

Momany, Y., Zaggia, S., Gilmore, G., Piotto, G., Carraro, G., Bedin, L. R., \& de Angeli, F. 2006, A\&A, 451, 515

Paczynski, B., \& Stanek, K. Z. 1998, ApJ, 494, L219 
Pohlen, M., Beckman, J. E., Hüttemeister, S., Knapen, J. H., Erwin, P., \& Dettmar, R.-J. 2004, Penetrating Bars Through Masks of Cosmic Dust, 319, 713

Reylé, C., Marshall, D. J., Robin, A. C., \& Schultheisé, M. 2009, A\&A, 495, 819

Rix, H.-W., \& Rieke, M. J. 1993, ApJ, 418, 123

Rix, H.-W., \& Zaritsky, D. 1995, ApJ, 447, 82

Robin, A. C., Creze, M., \& Mohan, V. 1992, ApJ, 400, L25

Robin, A. C., Reylé, C., Derrière, S., \& Picaud, S. 2003, A\&A, 409, 523

Ruphy, S., Robin, A. C., Epchtein, N., Copet, E., Bertin, E., Fouque, P., \& Guglielmo, F. 1996, A\&A, 313, L21

Saito, R., et al. 2010, The Messenger, 141, 24

Sale, S. E., et al. 2010, MNRAS, 402, 713

Schechter, P. L., Mateo, M., \& Saha, A. 1993, PASP, 105, 1342

Skrutskie, M. F., et al. 2006, AJ, 131, 1163

van der Kruit, P. C. 1979, A\&AS, 38, 15

van der Kruit, P. C. 2007, A\&A, 466, 883 

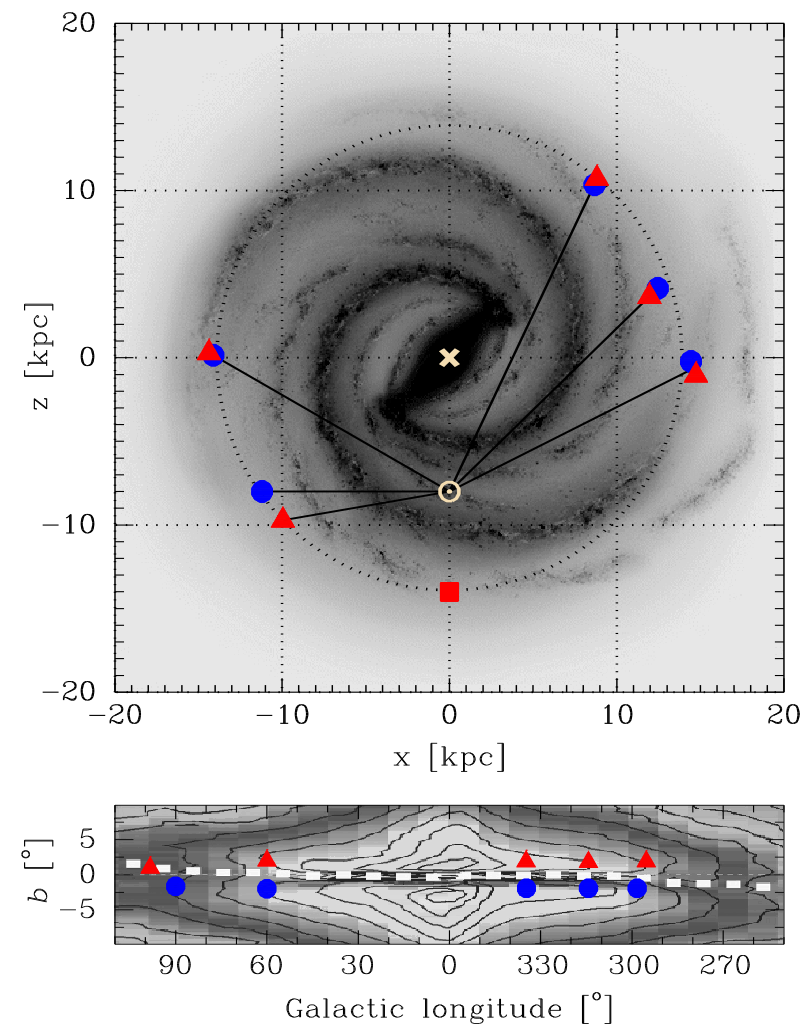

Fig. 3.- Measured positions of the edge of the Milky Way stellar disk. Top panel: Schematic face-on view of the Milky Way disk based on radio, infrared, and visible data (Churchwell et al. 2009), showing the positions of the edge of the stellar disk measured in different directions. The fields to the left are from the UKIDSS-GPS, the fields to the right are from the VVV survey, and the anticentre point at $R=14 \mathrm{kpc}$ is from (Robin et al. 2003; Reylé et al. 2009). We adopted a distance to the galactic centre of $R_{o}=8 \mathrm{kpc}$. This figure shows that a disk radius of $13.9 \pm 0.6 \mathrm{kpc}$ (dotted circle) fits the data very well. Bottom panel: Edge-on view of the Milky Way in Galactic $l, b$ coordinates with the position of the warped stellar disk marked with white squares (Momany et al. 2006). The positions of our fields are marked with triangles and circles, and the vertical scale has been stretched for clarity. 


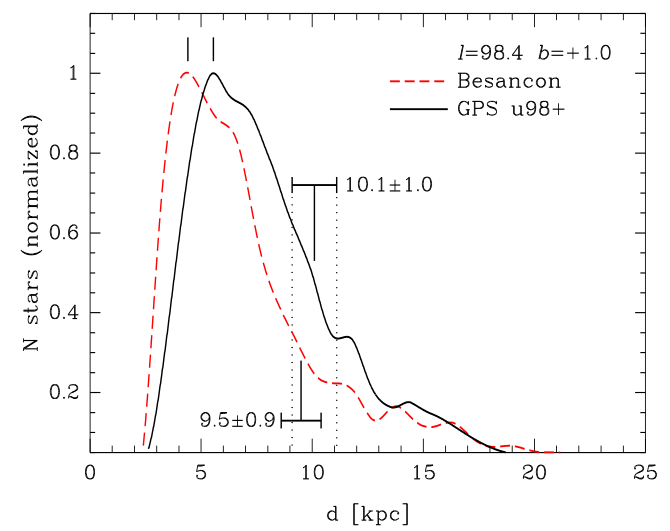

Fig. 4.- Comparison between data and model. Black solid line: Distance distribution for the field u098+ using UKIDSS-GPS data. Dashed red line: Simulation of the same field u098+ using the Besançon Galactic model with a scale length of $2.6 \mathrm{kpc}$ and an edge at $14 \mathrm{kpc}$. The model fits all features seen in the data distribution and the disk edge is detected at the expected place within the errors (see text). The distributions are arbitrarily normalized, and at shorter distances the discrepancy is due to photometric saturation. 